\title{
A TinyOS-Enabled MICA2-Based Wireless Neural Interface
}

\author{
Shahin Farshchi, Student Member, IEEE, Paul H. Nuyujukian, Aleksey Pesterev, Istvan Mody, and \\ Jack W. Judy*, Senior Member, IEEE
}

\begin{abstract}
Existing approaches used to develop compact low-power multichannel wireless neural recording systems range from creating custom-integrated circuits to assembling commercial-off-the-shelf (COTS) PC-based components. Custom-integrated-circuit designs yield extremely compact and low-power devices at the expense of high development and upgrade costs and turn-around times, while assembling COTS-PC-technology yields high performance at the expense of large system size and increased power consumption. To achieve a balance between implementing an ultra-compact custom-fabricated neural transceiver and assembling COTS-PC-technology, an overlay of a neural interface upon the TinyOS-based MICA2 platform is described. The system amplifies, digitally encodes, and transmits neural signals real-time at a rate of $9.6 \mathrm{kbps}$, while consuming less than $66 \mathrm{~mW}$ of power. The neural signals are received and forwarded to a client $P C$ over a serial connection. This data rate can be divided for recording on up to 6 channels, with a resolution of 8 bits/sample. This work demonstrates the strengths and limitations of the TinyOS-based sensor technology as a foundation for chronic remote biological monitoring applications and, thus, provides an opportunity to create a system that can leverage from the frequent networking and communications advancements being made by the global TinyOS-development community.
\end{abstract}

Index Terms-Brain-machine interface, EEG, epilepsy, smart dust, telemetry, TinyOS, wireless.

\section{INTRODUCTION}

$\mathbf{R}$ HYTHMICALLY VARYING electrical signals of large neural populations (i.e., field potentials) can vary slowly $(1 \mathrm{~Hz})$ or quickly $(100 \mathrm{~Hz})$. Such brain-wave activity has been correlated to specific physiological outcomes, such as sleep, excitation, and epilepsy. In order to quantify field-potential brain activity, an electroencephalogram (EEG) is recorded by measuring the potential difference between a pair of electrodes placed in or on the brain region of interest.

Although EEG recordings are frequently performed as acute experiments (e.g., $<6 \mathrm{~h}$ ), some studies require chronic or longer-term measurements. For example, the study of epilepsy requires continuous recordings to be made over a period of several days. Conventional EEG techniques use a direct-wired connection between the subject and the measurement tool.

Manuscript received February 14, 2005; revised January 22, 2006. Asterisk indicates corresponding author.

S. Farshchi, P. H. Nuyujukian, and A. Pesterev are with the Electrical Engineering Department, University of California, Los Angeles, CA 90095 USA (e-mail: shahin@ee.ucla.edu; herag@ee.ucla.edu; alexp@ee.ucla.edu).

I. Mody is with the Department of Neuroscience , University of California, Los Angeles, CA 90095 USA (e-mail: mody@ucla.edu).

*J. W. Judy is with the Electrical Engineering Department, University of California, Los Angeles, CA 90095 USA (e-mail: jack.judy@ucla.edu).

Digital Object Identifier 10.1109/TBME.2006.873760
Typically, this connection consists of a bundle of fine wires that can frequently limit animal behavior. In addition, the wired connection prevents the environment from containing natural elements such as tubes and tunnels. The constraints of such direct-wired connections have the potential for skewing the obtained results. A wireless recording system could be used to remove the aforementioned constraints. Such a wireless neural recording system must be capable of sensing, amplifying, and transmitting neural signals with a sampling frequency of at least $100 \mathrm{~Hz} / \mathrm{channel}$ (for reliably capturing EEG), while being small, low cost, lightweight, and low power. The system also requires a receiver to receive, demodulate, and display the transmitted neural signals.

Existing approaches to develop a wireless EEG measurement tool have ranged from designing a custom-microfabricated recording and telemetry system [1]-[3], to the use of commercial-off-the-shelf (COTS) PC technology [4]. Each approach has its own set of advantages and drawbacks. The advantages of custom designing each subsystem and component of a neural recording system include: 1) a greater degree of design flexibility; 2) the ability to optimize each subsystem in order to minimize power consumption, noise, and system dimensions. Such custom-designed systems can optionally be integrated in a hybrid CMOS-MEMS process [5] to yield neural probes with integrated data-acquisition/transmission systems in the interest of decreasing the overall size and increasing the signal-to-noise ratio. However, advances can only be incorporated with a very significant, costly, and time-intensive re-integration effort.

In contrast, designing a neural interface that uses mainstream PC-COTS-technology, such as a scaled-down PC motherboard with an 802.11b PCMCIA card, requires a smaller development effort to quickly yield a relatively high level of on-board digital signal processing and networking capabilities [4]. However, these systems are very bulky and power intensive, since they were not originally designed for truly compact low-power applications.

A novel approach would be a compromise between custom designing each subsystem and using PC-COTS components. This approach would achieve a balance between low-noise and low-power signal transmission, data communication, system size, and networking performance.

\section{EXISTING APPROACHES FOR DEVEloping NeURAL RECORDING TECHNOLOGIES}

Song [6] was among the first to introduce a fully integrated single-chip system for bio-data acquisition, digitization, and telemetry. The system consists of a custom-fabricated preamplifier and sigma-delta ADC converter that couples one of 
two ring oscillators, which are separated by $400 \mathrm{kHz}$, to the output via a multiplexer circuit to create an FSK signal. The system occupies a very small footprint $\left(2.5 \times 2.5 \mathrm{~mm}^{2}\right)$, and requires no off-chip components (except for a $2-\mathrm{cm}$ antenna and a battery) while consuming only $10 \mathrm{~mW}$ of power from an external $2.5-\mathrm{V}$ supply. The output signal center frequency can be adjusted from 5 to $56 \mathrm{MHz}$. Mohseni $e t a l$. at the University of Michigan have succeeded in demonstrating a custom-designed and microfabricated wireless multichannel recording system for neural-recording applications. The system [3] is capable of amplifying, multiplexing (TDMA), and transmitting seven channels of wideband ( $300 \mathrm{~Hz}$ to $4 \mathrm{kHz}$ ) neural signals over the FM band (tunable within 94 to $98 \mathrm{MHz}$ ). The system features a neural preamplifier circuit that provides ac amplification and dc baseline stabilization. Signals are received and demodulated by an FM receiver, and subsequently de-multiplexed, reconstructed, and displayed by a LabView-enabled PC. The system requires three passive external components (when operated with batteries) that can be wire bonded to the chip, thus yielding a very compact size $\left(2.2 \times 2.2 \mathrm{~cm}^{2}\right.$, excluding passive components) that weighs 1.1 grams with a 3-V battery attached. The system has a transmission range on the order of a few meters, and is capable of one-way communications while consuming $2.05 \mathrm{~mW}$ from the 3-V supply. This system is fabricated using an AMI double-poly double-metal $1.5-\mu \mathrm{m}$ n-well process. DiMichele [7] has designed and tested the individual blocks (preamplifier, filter, VCO and PA) of a 16-channel wireless neural transmitter that shares its basic design with that described by Mohseni [1]-[3]. The system consumes $3.8 \mathrm{~mA}$ from a 4.75-V supply.

Parramon [8] has reported an inductively powered implantable telemetry microsystem that measures $30 \mathrm{~mm}^{2}$ (excluding off-chip passive components) and consumes $24.5 \mathrm{~mA}$ of current. The system receives signals by modulating the amplitude of the inductive power supply, and transmits neural signals at a rate of up to $468 \mathrm{kbps}$ via FSK; however, the system was not fully functional at the time publication. Subsequent work by Irazoqui-Pastor [9] at the University of California, Los Angeles has successfully yielded an inductively powered single-channel wireless neural recording system. The implanted device amplifies, frequency modulates, and transmits a single channel of wideband neural signals to a spectrum analyzer, which demodulates the neural signals and feeds them via the Ethernet to a client PC (in a manner similar to [1]-[3] and [7]). The system is very compact $\left(5 \times 5 \times 10 \mathrm{~mm}^{3}\right)$ and weighs less than one gram. In addition, due to being inductively powered, this custom-integrated circuit (IC) wireless neural transmitter can remain implanted indefinitely for truly long-term chronic experiments. This system is fabricated using the TSMC $0.35-\mu \mathrm{m}$ CMOS process. This system provides one channel of neural signals over a 1-meter $(\mathrm{m})$ transmission range.

Researchers at Duke University have taken an entirely different approach to develop a wireless neural recording system [4]. Since their system was originally intended for use on owl monkeys instead of rodents, weight and power-consumption considerations were not as severe. Therefore, they decided to use existing COTS PC components to best leverage state-of-the-art signal-processing and communication technologies. The system is capable of acquiring, digitizing (with 12-bit resolution at a 32-kHz sampling rate), multiplexing, and transmitting 12 channels of neural signals to any $802.11 \mathrm{~b}$-enabled PC while consuming $4 \mathrm{~W}$ of power. The system is rather large $\left(5.1 \times 8.1 \times 12.4 \mathrm{~cm}^{3}\right)$, while weighing in at a substantial $235 \mathrm{~g}$ excluding the required lithium-ion batteries which last $67 \mathrm{~min}$ before needing to be removed and recharged.

A different COTS-based approach, chosen by researchers at Aachen University, consisted of attaching two discrete neural preamplifier circuits to the inputs of a commercial-off-the-shelf FM Stereo transmitter [10]. The system is capable of transmitting two channels of single-unit activity simultaneously, which are subsequently demodulated by a commercial FM Stereo receiver for analysis. The discrete nature of the system yields a package larger $\left(2.5 \times 1 \times 0.5 \mathrm{~cm}^{3}\right)$ than that of [1], which delivers similar performance. Researchers at the University of Tokyo have successfully developed a similar neural telemetry system for recording neural signals from insects [11]. A microelectrode is attached to the miniature circuit board $\left(1.5 \times 0.8 \mathrm{~cm}^{2}\right)$ via a polyimide ribbon cable. The neural preamplifier circuit is a COTS instrumentation amplifier (AD627) that provides a large input impedance for the neural signals of interest that are to be transmitted via a COTS FM transmitter. The system can transmit single-unit activity from insects to a COTS FM receiver for demodulation and subsequent analysis.

Cleveland Medical Devices has commercialized a product [12] in this space, but due to its proprietary nature, there are not many design details available. The device operates on the 902-MHz to $928-\mathrm{MHz}$ band and operates using frequency-shift keying (FSK). However, the requirement for a bulky $9-\mathrm{V}$ power supply and relatively large size $\left(6.4 \times 5.1 \times 1 \mathrm{~cm}^{3}\right)$ and weight ( $>68 \mathrm{~g}$ ) does imply extensive use of COTS components. The system is capable of acquiring, digitally encoding, and transmitting 8 channels of EEG signals, each of which are sampled at $250 \mathrm{~Hz}$. Once again, the system is only capable of one-way communication, although it does boast a relatively large transmission range of $46 \mathrm{~m}$.

\section{TinyOS And the Mica-Based Sensor Network}

Until recently, wireless devices consisted of relatively complex, expensive, and high-power systems, such as cell phones, PDAs, and wireless-enabled laptops that target specific and highly standardized applications and rely heavily on a powerful infrastructure (e.g., such as satellites, star-network base stations, etc.). Researchers at the University of California, Berkeley opted for a new approach in wireless-system design: one that involves low-cost embedded devices that can be implemented for a variety of applications [13]. This effort resulted in the development of the Mica platform: a self-configuring multihop (mesh) network platform for remotely monitoring distributed low-frequency phenomena [14]. This Mica platform operates on a component-based runtime environment called TinyOS (also developed at UC Berkeley) that has been specifically designed to provide support for deeply embedded systems with a minimal amount of physical hardware. Each node of this sensor network has sensing, communication, $\mathrm{I} / \mathrm{O}$, and processing capabilities, thus allowing it to act as a data router, sensor interface, and control point simultaneously. The TinyOS-enabled MICA2 platform grants 
high-level applications with direct and efficient control over low-level hardware. Consequentially, developers can optimize performance by implementing application-specific high-level networking and communications protocols, while controlling low-level hardware such as the radio and ADC. This programming, networking, and communication flexibility is in contrast to other mainstream wireless communications technologies that require the use of predefined protocols that cannot be tailored to specific applications (e.g., low-frequency, low-duty-cycle sensing for mesh network topographies versus wideband, real-time data acquisition for peer-to-peer communication).

The wireless sensor nodes, which are commonly referred to as "motes," have been designed to operate using TinyOS and are currently being used in wildfire-instrumentation, habitat-monitoring, and global-positioning applications, to mention just a few [15]-[17]. The data-throughput performance of the motes is severely constrained by their ultra-low-power operation and large-scale mesh networking capabilities. Custom data-acquisition and communication protocols could increase data throughput at the expense of increased power-consumption and mote-to-mote networking capabilities.

The two types of motes used in this work are the MICA2 and MICA2DOT motes produced by Crossbow Technology Inc. (San Jose, CA) [18]. A basic system schematic of the MICA2DOT mote is displayed in Fig. 1. The MICA2DOT has 6 input channels, each with its own 10-bit ADC. Data is processed by a microprocessor (Atmega128, Atmel Corp., San Jose, CA) with $512 \mathrm{kB}$ of flash memory. Data transmission to and from both the MICA2 and MICA2DOT is handled by a $916-\mathrm{MHz}$ radio (CC1000, Chipcon AS, Oslo, Norway) via FSK. An 8.2-cm-long, solid-copper-wire whip antenna is used on both the MICA2 and MICA2DOT motes. When the two $1.5-\mathrm{V}$ dry-cell batteries are installed, the MICA2 is approximately the size of a matchbox $\left(58 \times 32 \times 15 \mathrm{~mm}^{3}\right)$. The MICA2DOT uses essentially the same computational and communication hardware as the MICA2, but in a much smaller form-factor. The diameter of the MICA2DOT is roughly that of a United States quarter Dollar (25 mm), and its thickness is approximately $6 \mathrm{~mm}$. An MIB510 serial PC interface is used to allow the motes to communicate with a PC. The PC can be configured to broadcast sensor readings directly over its Internet connection, thus allowing any user over the Internet to access the sensor readings. Photographs of the MICA2, MICA2DOT, and MIB510 can be found at [18].

The work in this paper has been directed toward investigating new software and hardware designs that can be used to enable one or more motes to perform multichannel wireless neural recordings from several freely moving subjects simultaneously. Specifically, the goals of the work presented in this paper were to 1) implement an analog neural preamplifier circuit and interface it with the MICA2DOT mote, 2) develop a media-access-control (MAC) protocol to enable two MICA2-based motes to communicate at the bandwidth necessary to transmit and receive six channels of EEG, 3) design a TinyOS component that samples data from the analog input channels, encodes the samples in the form of discrete data packets, and transmits them over the radio, and 4) develop a client-side signal-reconstruction and display application.

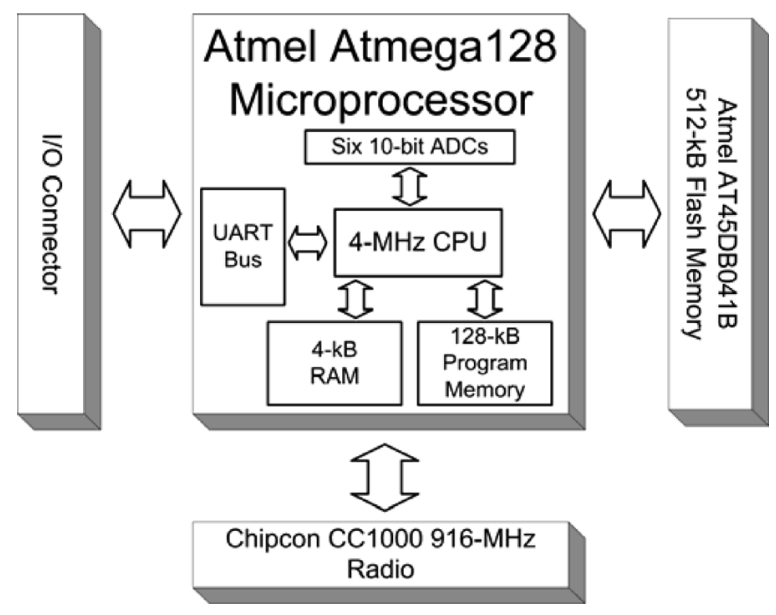

Fig. 1. System-level diagram of the MICA2DOT mote.

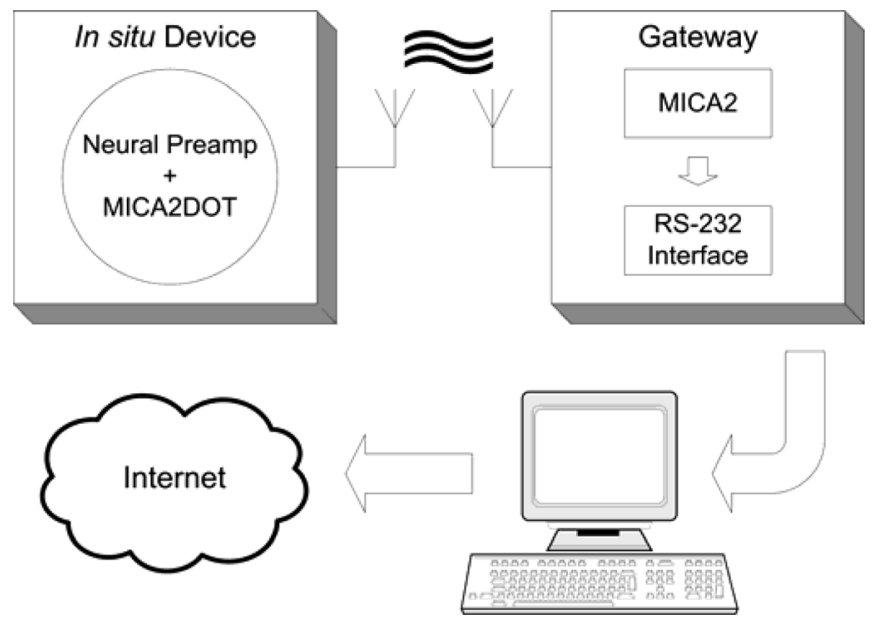

Fig. 2. Schematic diagram of the wireless neural recording system.

\section{System Design}

The overall system design can be divided into two major areas: hardware and software. A neural preamplifier circuit is required to properly amplify and level-shift the differential neural signals. TinyOS-software components are required to implement data-acquisition, signal-transmission, signal-reception, and wireless media-access protocols that achieve high data throughput. Finally, a client-side user interface is required to properly interpret, store, and display the received waveforms. A top-level diagram of the neural interface system is displayed in Fig. 2, and a photograph of complete neural recording system is displayed in Fig. 3.

\section{A. Hardware}

Each EEG channel is sensed differentially by a pair of implanted depth electrodes. A neural preamplifier circuit is used to take the differential signals and amplify, level-shift, and convert them into to a single-ended waveform ranging from $0 \mathrm{~V}$ to the MICA2DOT-battery voltage (nominally $3 \mathrm{~V}$ ) in order to be properly digitized by the MICA2DOT ADC. This preamplifier circuit has been designed to interface directly with the MICA2DOT mote. The heart of the neural preamplifier is an 


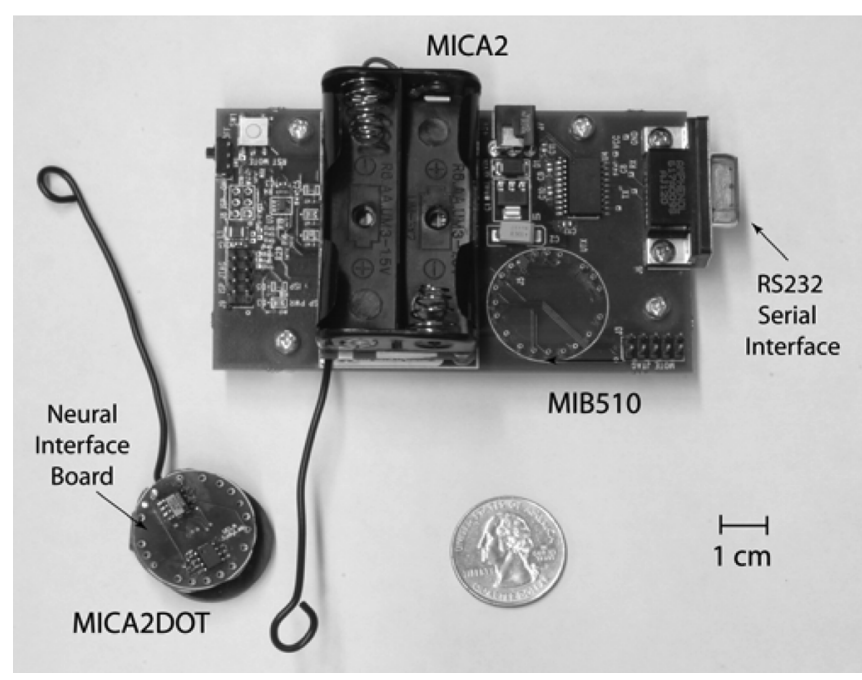

Fig. 3. Photograph of complete neural recording system.

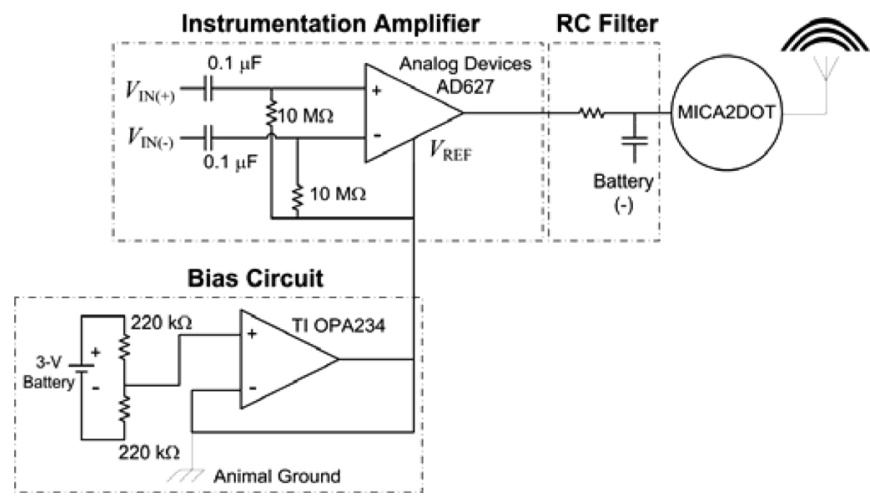

Fig. 4. Neural preamplifier circuit.

instrumentation amplifier (AD627, Analog Devices Inc., Norwood, MA). The gain of the AD627 is set by an external resistor to 200 , which yields a $3-\mathrm{V}$ peak-to-peak output for a $15-\mathrm{mV}$ peak-to-peak input signal. This corresponds to a fixed maximum resolution of approximately $59 \mu \mathrm{V}$. The output has been referenced to half the supply voltage by a simple resistive divider followed by an op-amp (OPA234, Texas Instruments Inc., Dallas, TX) configured as a voltage follower (to allow the use of a single coin cell). To avoid high-frequency noise from being aliased into the sampled signal, the AD627 output is followed by a simple RC-filter. This circuit consumes less than $3 \mathrm{~mW}$ of power from a single $3-\mathrm{V}$ coin cell. A circuit-level schematic of the neural preamplifier circuit is displayed in Fig. 4.

\section{B. Software}

TinyOS-software components have been written to implement data-acquisition and wireless MAC protocols for the MICA2DOT transmitter. The MICA2 receiver operates on a standard TinyOS component to receive packets and broadcast them via a serial port to a PC. The MICA2DOT operates on a custom data-acquisition component. The Chipcon CC1000 radio is set to operate at its maximum data rate $(38.4 \mathrm{kbps}$ for the MICA2DOT, though the CC1000 can operate at $76.8 \mathrm{kbps}$ on the larger-form-factor MICA2), and Manchester encoding is used for simple error recognition by encoding each logical "1" as " 01 " and each logical " 0 " as "10." This encoding brings the available raw bandwidth down to $19.2 \mathrm{kbps}$ for the MICA2DOT. The radio copies data from a buffer on the Atmel microprocessor to its send register on a per-byte basis. This copy event is invoked by the internal clock of the radio, which runs at a frequency equivalent to the radio transmit speed in terms of bytes/s (i.e., $2.4 \mathrm{kHz}$ for a radio transmit speed of 19.2 kbps with Manchester encoding).

The TinyOS operating system handles processes sequentially on a per-task basis. The tasks are processed in the order in which they are placed in the task queue. The inability to prioritize any single task prohibits the scheduling of precise time-sensitive tasks (e.g., ADC sampling) in software. ADC-sampling delays lead to nonuniform sampling intervals (i.e., sampling jitter), which result in significant distortion. An alternative would be to invoke a hardware-based timer that would periodically issue an interrupt to the processor. Unfortunately, setting a hardwarebased timer to sample the ADC at arbitrary periods results in packets being dropped at the receiver end when the radio operates at 19.2 kbps. Packet loss results in nonrecoverable data loss. However, synchronizing data acquisition with radio communication greatly reduces this loss.

To minimize sampling jitter and data loss, the data-acquisition component prompts the ADCs to sample data once per two radio timer periods [i.e., $1.2 \mathrm{kHz}$ to accommodate for the packet header and cyclic redundancy check (CRC) bytes], digitize it in the form of a 10-bit integer value, and store the 8 most significant bits in a buffer. When 40 readings are taken, a packet header indicating the source mote ID, packet size, final reading ID number (for time referencing), and CRC bytes are copied to a buffer for subsequent transmission over the radio. Data resolution can be increased to a maximum of 10-bit (as dictated by the ADC); however, ADC sampling speed must be reduced proportionally to compensate for the increased signal resolution, thus resulting in a lower data throughput. The parsing algorithm on the client must also be designed to properly account for then length of each word that corresponds to a data point. Fig. 5 illustrates a timing diagram for data acquisition, transmission, reception, and forwarding to a client PC with a MICA2 sampling transmitting at 2400 samples/s. By examining the top waveform in Fig. 5, one will notice that sampling periods are slightly skewed when the radio starts and stops transmitting packets, which could be an indication of a sampling event being preempted by the radio-send process execution and termination.

The MAC protocols, originally developed for the MICA2 platform, were designed to facilitate multihop communication among large (i.e., hundreds) of individual sensor nodes in an unregulated frequency band. This large-scale networking capability came at the expense of decreased data throughput among individual sensor nodes, due to the sensor nodes having to wait for a random period of time before transmitting a packet. In this work, a new MAC protocol was developed that instructs the transmitter to send whenever the radio-transmit buffer is full. This modification eliminates the ability of the mote to receive packets from the base station, even though it is equipped with a receiver. For comparison, using the standard software, experimental bench testing yielded an effective data rate of less 


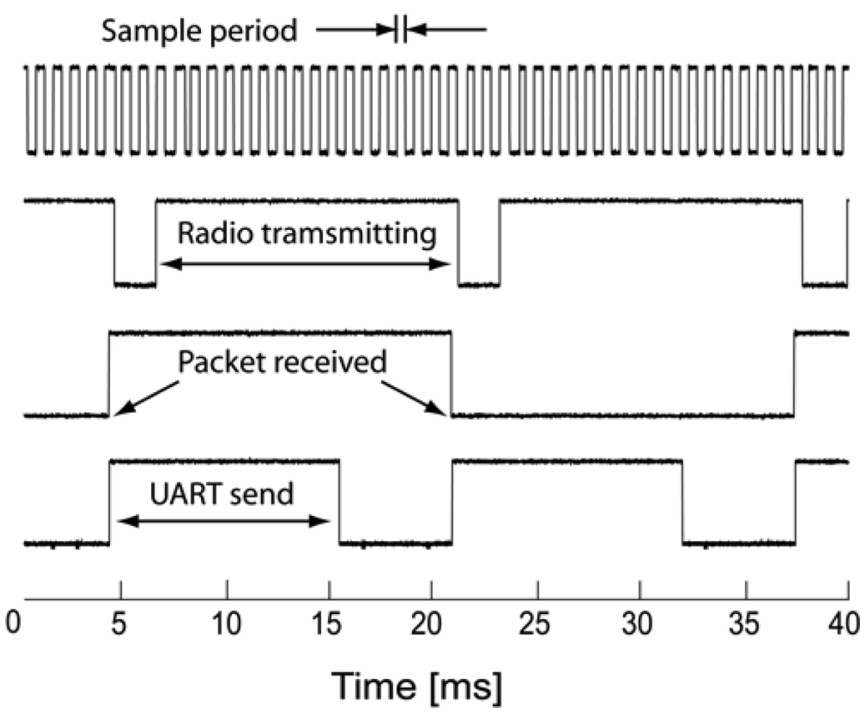

Fig. 5. Timing diagram illustrating sampling events with respect to data transmission and reception. The transitions of the top and second waveforms indicate data-sampling and radio-transmission start/stop events from the transmitting mote, respectively. The transitions of the third and fourth waveforms from the top indicate packet reception, and UART (serial) send start/stop times on behalf of the receiver, respectively. The third waveform indicates that a packet is received as soon as the radio stops transmitting, while the bottom waveform indicates that the receiver begins transmitting the packet to the $\mathrm{PC}$ as soon as it is received via the UART connection.

than 700 bps. By removing the radio wait time before transmitting a packet, we achieved an effective data rate of $3.84 \mathrm{kbps}$. By synchronizing ADC signal acquisition with the processor buffer-to-radio data transfer, we achieved a data rate of $9.6 \mathrm{kbps}$ with the MICA2DOT. The MICA2 radio can be set to operate at $76.8 \mathrm{kbps}$ (as opposed to $38.4 \mathrm{kbps}$ on the MICA2DOT); therefore, in applications where the added size of the MICA2 is not of concern, the user can obtain double the data throughput of the smaller MICA2DOT (i.e., 19.2 kbps) using the same methods described above.

To maximize the recordable neural-signal bandwidth, given our achieved data-throughput performance, we implemented a Java-based client-side signal-reconstruction program. This program uses digital signal processing to interpolate points in between sampled points to achieve a tenfold improvement in signal resolution (although it does not improve signal bandwidth). The algorithm applies an eighth-order Chebyshev filter (with a 90\%-settling time of approximately 55 samples and a cut-off frequency at half the original sampling frequency) to an up-sampled (by a factor of 10) version of the original sampled neural signal. The results allow the neural activity (within the Nyquist band, which is determined by the original sampling frequency) occurring between the sampled points to be deduced by using the frequency-domain information encapsulated in the original sampled signal [19].

\section{EXPERIMENTAL TESTING}

Experimental testing was performed in two categories: bench testing and in-situ testing. Bench testing was performed to assess the specific performance metrics of the system, such as data rate, range, power consumption, and signal resolution. In situ testing was used to evaluate the overall performance of the system in its respective application environment.

\section{A. Bench Test of System}

The gain-bandwidth characteristics of the preamplifier circuit were tested in saline by applying a sinusoid to the saline solution and measuring the gain between the amplifier output leads and the input leads from the saline solution. The amplifier provides a gain of 200 at frequencies ranging from a few $\mathrm{Hz}$ to $1 \mathrm{kHz}$. Note that the anti-aliasing RC filter is omitted in this test, as it is tuned to the sampling frequency of the transmitter.

To assess the performance of the neural preamplifier circuit, as well as the total bandwidth of the data-acquisition and transmission system, an extracellular neural recording dataset was used. The data was originally acquired in vivo from freely moving rats using five four-channel MOSFET input operational amplifiers mounted in the cable connector to remove movement artifacts. Data were recorded wide band $(0.1 \mathrm{~Hz}$ to $5 \mathrm{kHz})$ and sampled at $10 \mathrm{kHz} /$ channel (16 channels) with 12-bit precision. The dataset was programmed into an arbitrary waveform generator (HP 33120A, Agilent Technologies Inc., Palo Alto, CA) whose outputs were applied to saline. The original waveform programmed into the HP $33120 \mathrm{~A}$, the signal from saline, and the received signal (sampled at 2400 Samples/sec) are depicted in Fig. 6. To demonstrate the usefulness of the system for EKG recording, a 5-mV simulated cardiac signal from the HP $33120 \mathrm{~A}$ was applied to the preamplifier with respect to ground (Fig. 7) and sampled at 480 samples/s, while the other channels were left open. When recording from saline, the smallest detectable signal amplitude is on the order of several $\mathrm{mV}$ (due to background noise); however, provided that there is no noise, the $3-\mathrm{V}$ dynamic range, 8-bit resolution, and gain of 200 implies that a $59-\mu \mathrm{V}$ signal can be detected in the presence of no noise from the preamplifier circuit or the environment. Signals that are output from the preamplifier circuits are multiplexed and sampled sequentially by the single ADC on the Atmel Atmega 128L microprocessor. This successive acquisition method results in no noticeable signal crosstalk subsequent to digitization. However, even if sufficient measures are taken in creating ground planes on the preamplifier circuit board, there is a possibility for the signals to be coupled together at the electrode level, which is dependant on electrode and preamplifier placement.

To measure the effect of sampling jitter on signal distortion, a pure $200-\mathrm{Hz}$ tone was sampled by one of the input channels at a rate of $1.2 \mathrm{kHz}$ (to model 2-channel operation). The energy of the first and second harmonics were measured against that of the fundamental frequency, which yielded a worst case total harmonic distortion of $1.7 \%$.

The range of the neural transmitter was tested in a noisy laboratory environment equipped with monitors, oscilloscopes, microwaves, IEEE 802.11b transceivers, and a refrigerator. The system data-loss rate was assessed by measuring the total number of packets lost/2500 transmitted (which corresponds to approximately 100000 data points). The measurements were taken with two MICA2 motes each equipped with an 8.2-cm-long solid-copper-wire whip antenna. As shown in Fig. 8, the rate of packet loss is relatively constant over a 9-m 


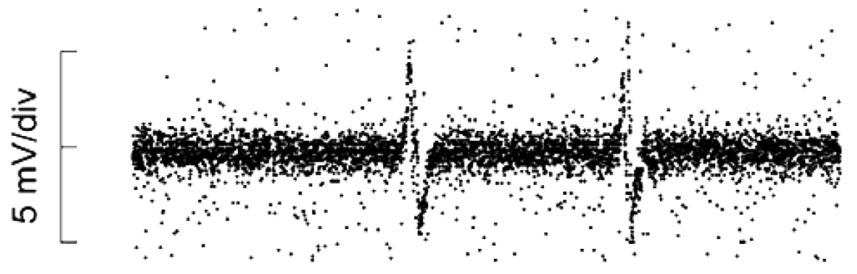

(a) Input to preamplifier from saline

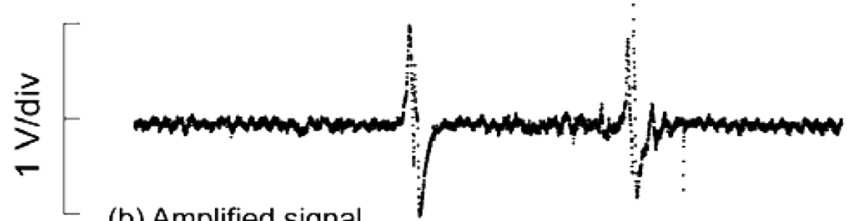

(b) Amplified signal

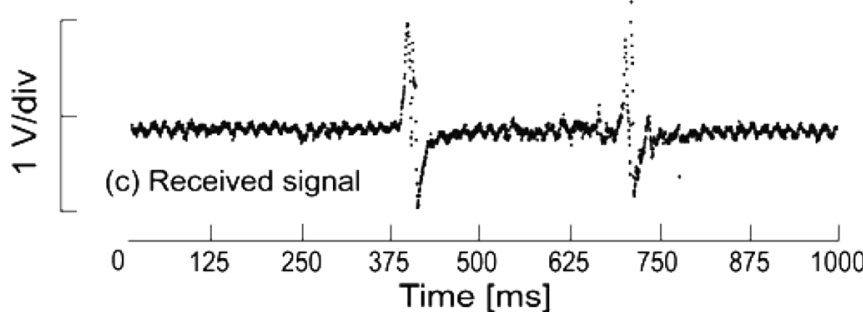

Fig. 6. Simulated input, amplified, and received EEGs recorded from saline.

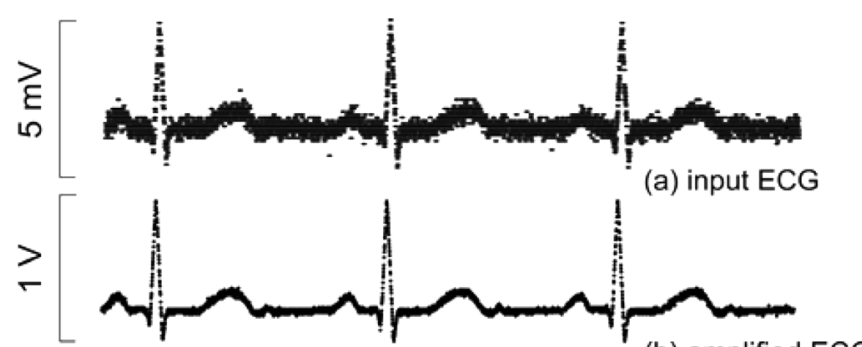

(b) amplified ECG

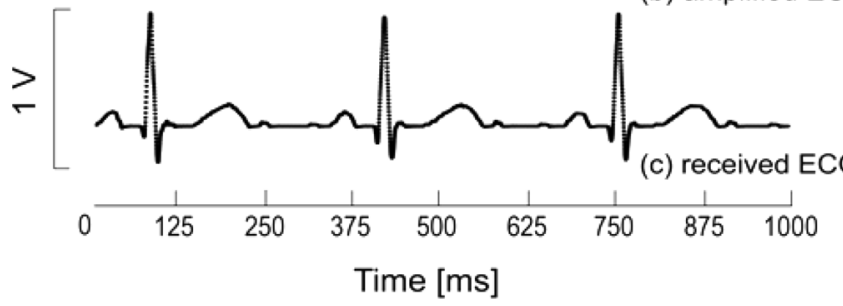

Fig. 7. Input, amplified, and received/reconstructed 3-Hz ECG.

range. At larger distances, the data rate is highly affected by antenna orientation and line-of-sight, which is unacceptable in an application where readings are to be taken with moving and interacting test subjects. Intermittent packet loss due to moving objects between the transmitter and receiver, and changes in antenna orientation is negligible within a 2-m range; therefore, the ideal operating range for moving test objects in an environment with obstacles and other animals is two meters.

Power consumption was measured at $66 \mathrm{~mW}$ from a $3-\mathrm{V}$ cell. With two 1.5-V alkaline batteries (Panasonic Industrial, Secaucus, $\mathrm{NJ}$ ), the system lasts for approximately $100 \mathrm{~h}$ before requiring new batteries. A single $3-\mathrm{V}$ cell (2L76, Energizer, Milford, CT) lasts for approximately $5 \mathrm{~h}$.

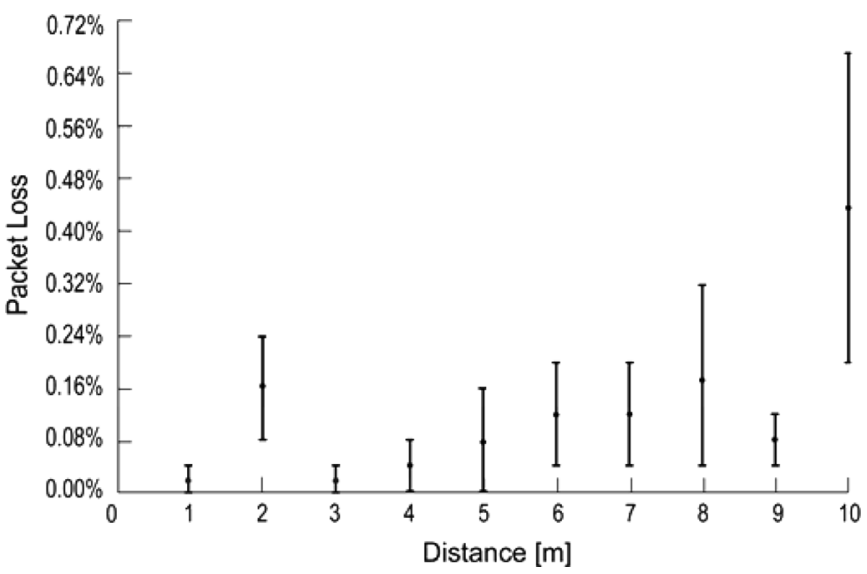

Fig. 8. Data loss as a function of distance. Data loss beyond 9 meters is a function of antenna orientation and requires line-of-sight, which is unacceptable in this application.

\section{B. Animal Testing}

The system was tested on a live rat in a typical laboratory environment. An adult C57/B16 mouse (Harlan, Indianapolis, IN) was anesthetized with $100 \mathrm{mg} / \mathrm{kg}$ ketamine, $5.2 \mathrm{mg} / \mathrm{kg}$ xylazine, and $1.0 \mathrm{mg} / \mathrm{kg}$ acepromazine according to a protocol approved by the UCLA Chancellor's Animal Research Committee. A lengthwise incision was made along the scalp and a small burr hole was made $2.2 \mathrm{~mm}$ posterior to bregma and $1.7 \mathrm{~mm}$ lateral to the midline. A micromanipulator was used to place a hippocampal depth electrode (Plastics One, Roanoke, VA) in the hippocampus at a depth of $2.0 \mathrm{~mm}$. The electrode was fixed to the skull using dental cement and the animal was allowed to recover for $48 \mathrm{~h}$ before recording was performed. The animal was anesthetized again prior to performing the experiment. The implanted depth electrodes were attached to the preamplifier circuit which was interfaced with a MICA2 mote. A lead from the skull was tied into the AD627 reference terminal, which was set to half the supply voltage. To model temporal-lobe epilepsy, the animal was subsequently injected with kainic acid at $15 \mathrm{mg} / \mathrm{kg}$ [20]. Recordings were taken differentially at a rate of 480 samples/s on one of the 6 input channels. The $\mathrm{R}$ and $\mathrm{C}$ values for the anti-aliasing filter were $39 \mathrm{k} \Omega$ and $0.1 \mu \mathrm{F}$, respectively. These readings were transmitted, received, and reconstructed by a client PC. Fig. 9 depicts received and reconstructed recordings taken from the living rodent before and after kainic acid injection for observing normal and seizure EEGs.

Unfortunately, the extremely low amplitude of the anesthetized animal's EEGs resulted in the system only capturing the peaks of neural activity until the animal began experiencing seizures. This explains the ringing that is present in the reconstructed waveforms depicted in Fig. 9. In addition, an improper ground connection resulted in the DC bias that is present in the recorded signals.

\section{CONCLUSION}

In this paper, we have demonstrated the design of a TinyOS-based wireless neural interface. The transmitter is a composed of a MICA2DOT mote (commercially available 
TABLE I

WireleSS Microsystem PERFORMANCE SUMMARY (ADAPTED From [1])

\begin{tabular}{|c|c|c|c|c|c|c|c|}
\hline Institution & UCLA & Michigan & Duke & UCLA & Aachen & Tokyo U. & Clev. Med. \\
\hline Number of Data Channels & 6 & 7 & 12 & 1 & 2 & 1 & 8 \\
\hline Telemetry Link Freq (MHz) & 916 & $94-98$ & 2400 & 3200 & $88-108$ & $80-90$ & $902-928$ \\
\hline Communication Scheme & MCFSK & TDMA/Analog FM & $802.11 \mathrm{~b}$ & Analog FM & FM Stereo & Analog FM & FSK \\
\hline Power Supply & $3 \mathrm{~V}$ & $\pm 1.5 \mathrm{~V}$ & $3.3 \mathrm{~V}$ and $5 \mathrm{~V}$ & Inductive & $\pm 1.4 \mathrm{~V}$ & $3 \mathrm{~V}$ & $9 \mathrm{~V}$ \\
\hline Max. Power Dissipation & $66 \mathrm{~mW}$ & $\sim 2.05 \mathrm{~mW}$ & $4.0 \mathrm{~W}$ & $13.8 \mathrm{~mW}$ & - & $10 \mathrm{~mW}$ & - \\
\hline Transmission Range & $9 \mathrm{~meters}$ & a few meters & $9 \mathrm{~m}$ & $<1 \mathrm{~meter}$ & a few meters & $\sim 16 \mathrm{~m}$ & $<46 \mathrm{~m}$ \\
\hline Sensitivity & $0.06-15 \mathrm{mV}$ & $0.1-5 \mathrm{mV}$ & - & $0.015-15 \mathrm{mV}$ & - & - & - \\
\hline Total Bandwidth & $600 \mathrm{~Hz}$ & $10 \mathrm{kHz}$ & - & $10 \mathrm{kHz}$ & - & - & - \\
\hline Dimensions (cm) & $2.5 \times 2.5 \times 1$ & $0.22 \times 0.22$ & $5.1 \times 8.1 \times 12.4$ & $0.5 \times 0.5 \times 1$ & $2.5 \times 1 \times 0.5$ & $1.5 \times 0.8$ & $6.4 \times 5.1 \times 1$ \\
\hline Total Weight (w/ battery) & $12.8 \mathrm{~g}$ & $1.1 \mathrm{~g}$ & $235 \mathrm{~g}$ & $<1 \mathrm{~g}$ & $>3.1 \mathrm{~g}$ & $>0.1 \mathrm{~g}$ & $>68 \mathrm{~g}$ \\
\hline Reference & This Work & {$[3]$} & {$[4]$} & {$[9]$} & {$[10]$} & {$[11]$} & {$[12]$} \\
\hline
\end{tabular}

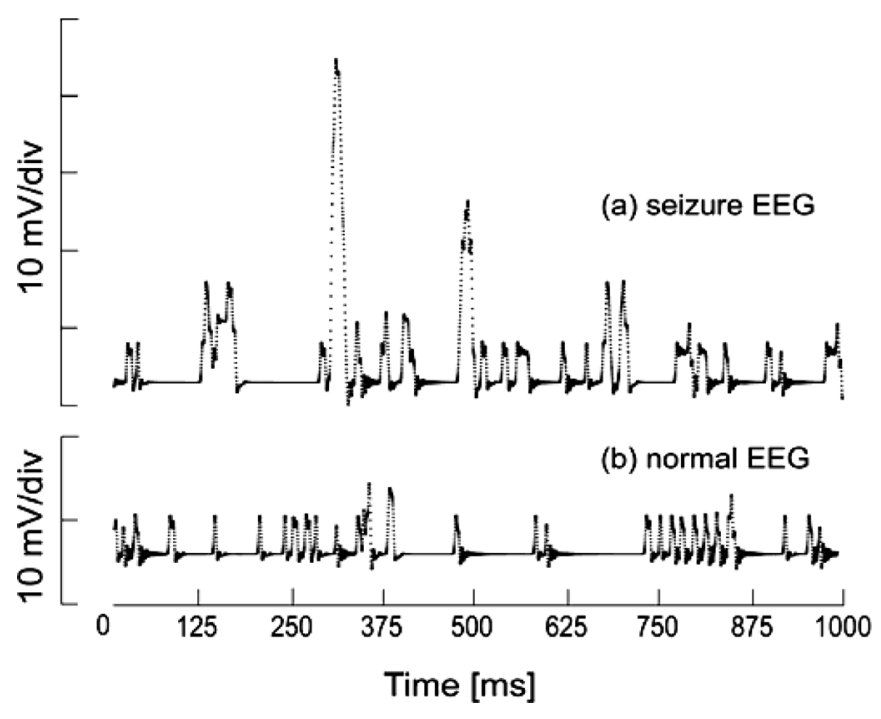

Fig. 9. Neural activity recorded in vivo.

from Crossbow Technology Inc. [18]) interfaced with a custom discrete neural preamplifier circuit that consists of a Texas Instruments OPA234, an Analog Devices AD627 instrumentation amplifier, and seven passive components/channel. A MICA2 mote interfaced with an MIB510 gateway (both of which are commercially available from Crossbow Technology Inc. [18]) receives and relays the signals to a client PC via a serial connection. The system operates on custom signal acquisition, transmission, archiving, and hosting software for wirelessly sensing, storing, and browsing recorded neural signals. Efforts are being made to commercialize the custom neural preamplifier circuit and software to make the technology accessible to brain researchers [21].

The system is capable of amplifying, sampling, transmitting, and reconstructing input signals at a rate of 12008 -bit samples/s. This data rate allows for the reliable transmission of up to $6 \mathrm{EEG}$ channels (with sampling rate of $200 \mathrm{~Hz} / \mathrm{channel}$ ), simultaneously from live, mobile test subjects. We have tested the system with synthesized neural signals from saline, and a live rodent. Table I illustrates the performance metrics of this technology, in comparison with other reported technologies. The following issues were encountered during the development of this system with and could severely handicap the neural interface on which it is based.

\section{A. Inability to Prioritize Tasks}

The TinyOS release used in this work (version 1.1.3) does not give the programmer the ability to prioritize precise time-sensitive tasks, such as data acquisition. In contrast, tasks are executed by the order in which they are placed in a task queue. Therefore, a task generated by hardware (i.e., a buffer copy command) could easily preempt a data-acquisition task. Delayed data-acquisition tasks result in sampling jitter (as illustrated in Fig. 5), which in turn result in signal distortion on the receiver end, since the signal-reconstruction program assumes that the time lapse between sample periods are identical and equal to the programmed sampling period. Simply attaching a timestamp to each sampled data point requires excessive bandwidth overhead.

\section{B. Requirement for Synchronous Data-Acquisition and Transmission}

The factor that limits the sampling rate of the MICA2 to 2.4 $\mathrm{kHz}$ (rather than the $4 \mathrm{kHz}$ that can be theoretically provided by the hardware) is due to the requirement for synchronous data acquisition and transmission. This maximizes the analog-to-digital converter (ADC) sampling rate to half the radio byte-copy period when taking 8-bit samples. Using a radio chip that does not copy the send buffer on a timed per-byte basis will allow the developer to set the data-acquisition period to a value independent of the radio speed. Crossbow Technology Inc. [8] has recently released a new class of motes called the MICAz. These motes use the same Atmel Atmega 128 processor, with a ZigBee-compliant (IEEE 802.15.4) transceiver that operates at $2.4 \mathrm{GHz}$ (the Chipcon CC2420). The processor only needs to dump to the radio-send buffer once every send cycle, eliminating the synchronization requirement that limits the MICA2. The MICAz may also not be as sensitive to antenna orientation and may not require line-of-sight. The antenna can also measure at less than an inch (as opposed to the two-inch whip antennas used in this work). Finally, the faster radio on the MICAz (256 kbps) allows the mote to receive messages while sampling and transmitting at high data rates. 


\section{Power Consumption Tradeoffs}

The data loss due to line-of-sight obstructions and antenna orientation becomes more pronounced as the radio power output is decreased and the distance between two MICA2-based motes is increased. In order to insure that the data loss was insignificant over a 2-m range, and independent of antenna orientation, the radio transmitter power was set to its maximum setting (10 $\mathrm{dBm})$. Consequently, the overall power consumption was quite high (i.e., $66 \mathrm{~mW}$ ). Using a radio that does not have these limitations would enable the developer to lower the radio output power level considerably for short-range applications.

This work demonstrates that the TinyOS-enabled MICA2 platform can be used as a foundation for low-power real-time wireless biosignal recording system if 1) time-critical tasks could be prioritized, and 2) data acquisition and transmission need not be synchronized. In addition, using a mote equipped with a transceiver that does not require line-of-sight and is not sensitive to antenna orientation, could allow the developer to set the radio output power to a lower level for short-range communication (i.e., on the order of a meter). Such adjustments could yield greatly reduced power consumption.

\section{ACKNOWLEDGMENT}

The authors would like to thank Dr. A. Bragin for providing neural recordings for their bench tests, and Dr. J. Maguire for preparing and performing the live animal experiments. They would also like to thank M. Rahimi, T. Stathopoulos, and L. Girod for their valuable advice. This paper is based upon work supported by the National Science Foundation (NSF) under Grant No. 0456125.

\section{REFERENCES}

[1] P. Mohseni and K. Najafi, "A wireless FM multichannel microsystem for biomedical neural recording applications," in Proc. IEEE Southwest Symp. Mixed Signal Design, Tucson, AZ, Feb. 23-25, 2003, pp. 217-222.

[2] — "Wireless multichannel biopotential recording using an integrated FM telemetry circuit," in Proc. 26th Annu. Int. Conf. Engineering in Medicine and Biology Soc., San Francisco, CA, Sep. 1-4, 2004, pp. 4083-4086.

[3] _ "A battery-powered 8-channel wireless FM IC for biopotential recording applications," in Dig. Tech. Papers 2005 IEEE Int. SolidState Circuits Conf., San Francisco, CA, Feb. 6-10, 2005, pp. 560-561.

[4] I. Obeid, M. A. L. Nicolelis, and P. D. Wolf, "A multichannel telemetry system for single unit neural recordings," J. Neurosci. Meth., vol. 133, pp. 123-135, Feb. 2004

[5] K. D. Wise, D. J. Anderson, J. F. Hetke, D. R. Kipke, and K. Najafi, "Wireless implantable microsystems: high-density electronic interfaces to the nervous system," Proc. IEEE, vol. 92, no. 1, pp. 76-97, Jan. 2004.

[6] H. J. Song, D. R. Allee, and K. T. Speed, "Single chip system for bio-data acquisition, digitization and telemetry," in Proc. 1997 IEEE Int. Symp. Circuits Systems, Hong Kong, Jun. 9-12, 1997, vol. 3, pp. 1848-1851.

[7] G. A. DeMichele and P. R. Troyk, "Integrated multichannel wireless biotelemetry system," in Proc. 25th Int. IEEE-EMBS Conf., Cancun, Mexico, Sep. 17-21, 2003, pp. 3372-3375.

[8] J. Parramon, P. Doguet, D. Martin, M. Verleyssen, R. Munoz, L. Leija, and E. Valderrama, "ASIC-based batteryless implantable telemetry microsystem for recording purposes," in Proc. 19th Int. IEEE-EMBS Conf, Chicago, IL, Oct. 30-Nov. 2 1997, pp. 2225-2228.
[9] P. Irazoqui-Pastor, I. Mody, and J. W. Judy, "Transcutaneous RF-powered neural recording device," in Proc. 24th Annu. Conf./Annu. Fall Meeting Biomedical Engineering Society, Houston, TX, Oct. 23-26, 2002, vol. 3, pp. 2105-2106.

[10] A. Nieder, "Miniature stereo radio transmitter for simultaneous recording of multiple single-neuron signals from behaving owls," $J$. Neurosci. Meth., vol. 101, pp. 157-164, Sep. 2000.

[11] S. Takeuchi and I. Shimoyama, "A radio-telemetry system with a shape memory alloy microelectrode for neural recording of freely moving insects," IEEE Trans. Biomed. Eng., vol. 51, no. 1, pp. 133-137, Jan. 2004.

[12] M. Modarreszadeh and R. N. Schmidt, "Wireless, 32-channel, EEG and epilepsy monitoring system," in Proc. 19th Int. Conf. IEEE/EMBS, Chicago, IL, Oct. 30-Nov. 2 1997, pp. 1157-1160.

[13] J. L. Hill and D. E. Culler, "Mica: a wireless platform for deeply embedded networks," IEEE Micro, vol. 22, no. 6, pp. 12-24, Nov./Dec. 2002.

[14] J. L. Hill, "System architecture for wireless sensor networks," Ph.D. dissertation, Dept. Comput. Sci., Univ. California, Berkeley, 2003.

[15] M. M. Chen, C. Majidi, D. M. Doolin, S. Glaser, and N. Sitar, "Design and construction of a wildfire instrumentation system using networked sensors (poster)," presented at the Network Embedded Systems Technology (NEST) Retreat, Oakland, CA, Jun. 17-18, 2003.

[16] J. J. Polastre, "Design and implementation of wireless sensor networks for habitat monitoring," M.S. thesis, Dept. Comput. Sci., Univ. California, Berkeley, 2003.

[17] D. Niculescu and B. Nath, "DV based positioning in ad hoc networks," Kluwer J. Telecommun. Syst., vol. 22, pp. 267-280, Jan. 2003.

[18] Crossbow Technology, Inc. [Online]. Available: http://www.xbow.com

[19] A. V. Oppenheim and R. W. Schafer, Digital Signal Processing, 1st ed. Upper Saddle River, NJ: Prentice Hall, 1989, pp. 87-91.

[20] A. Bragin, J. Engel, C. L. Wilson, I. Fried, and G. W. Mathern, "Hippocampal and entorhinal cortex high-frequency oscillations (100-500 hz) in human epileptic brain and kainia acid-treated rates with chronic seizures," Epilepsia, vol. 40, pp. 127-137, Feb. 1999.

[21] Vista Integrated Systems [Online]. Available: http://www.visys.biz

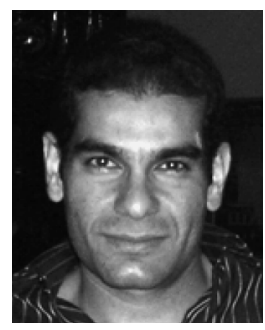

Shahin Farshchi (S'03) was born in Berkeley, CA, in 1978. He received the B.S. in electrical engineering and computer science at the University of California, Berkeley, with College of Engineering Honors in 2002, and M.S. in electrical engineering at the University of California, Los Angeles, in 2005. $\mathrm{He}$ is currently working toward the Ph.D. degree in electrical engineering at the University of California, Los Angeles.

His research interests include the design and implementation of fully integrated neural preamplifier circuits and embedded wireless biological monitoring systems.

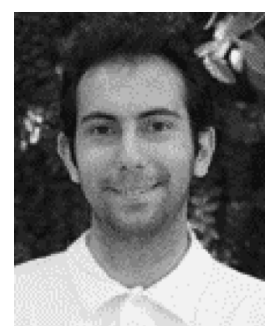

Paul H. Nuyujukian is currently working towards the B.S. degree in cybernetics at the University of California, Los Angeles.

He joined Prof. J. W. Judy's research group in January, 2004.

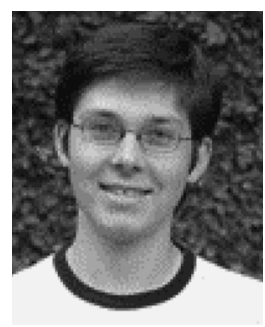

Aleksey Pesterev is currently working towards the B.S. degree in computer science with the electrical engineering option at the University of California, Los Angeles.

He joined Prof. J. W. Judy's research group in September, 2003. 


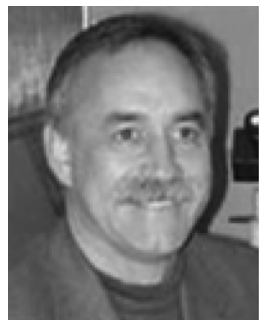

Istvan Mody received the Ph.D. degree in physiology (neurophysiology) from the University of British Columbia, Vanouver, BC, Canada. With an Izaak Walton Killam and a Canadian MRC Fellowhip; he started his postdoctoral studies at the Max-Planck Institute in Munich, Germany, and completed them at the Playfair Neuroscience Center at the University of Toronto. His first faculty appointment was in the Department of Neurology and Neurological Sciences at Stanford University, California.

He is the Tony Coelho Professor of Neurology and Professor of Physiology at the David Geffen School of Medicine at the University of California, Los Angeles. His research interests include ligand-gated ion channels, inhibition in the brain, basic mechanisms of epilepsies, and the regulation of intraneural calcium.

Dr. Mody's research on basic mechanisms of epilepsies has earned him several awards, including the Alfred Hauptmann Prize, the Michael Prize, and the Basic Scientist Award from the AES/Milken Foundation. He is a member of the Hungarian Academy of Sciences and a recipient of the Javits Award from the National Institutes of Health/NINDS.

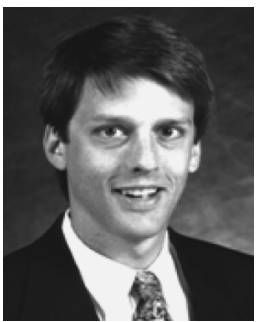

Jack W. Judy (S'87-M'96-SM'02) received the B.S.E.E. degree, with summa cum laude honors, from the University of Minnesota, Minneapolis, in 1989, and the M.S. and Ph.D. degrees in electrical engineering from the University of California, Berkeley, in 1994 and 1996, respectively. In his doctoral research he developed a ferromagnetic microactuator technology that is useful for a variety of applications, including optical, RF, and biomedical MEMS.

After graduating he worked for Silicon Light Machines, Inc., Sunnyvale, CA, an optical-MEMS start-up company commercializing a novel projection-display technology, from 1996 to 1997. He has been on the faculty of the Electrical Engineering Department at the University of California, Los Angeles, since 1997, where he is currently an Associate Professor. At UCLA, he is the Chair of the MEMS and Nanotechnology Major Field of the Electrical Engineering Department and the Director of the UCLA NeuroEngineering Training Program, which is a NSF-funded IGERT program sponsored jointly by the Biomedical Engineering and Neuroscience Interdepartmental Programs and the Brain Research Institute (BRI). He also serves as the Director of the UCLA Nanoelectronics Research Facility, a 10000 -sq.ft. clean room dedicated for research, and the Director of the UCLA Microfabrication Laboratory, a 3300-sq.ft. clean room dedicated for training students in the process of micromachining MEMS and integrated circuits. His research interests are primarily in the fields of MEMS and neuroengineering. His specific projects of interest include novel ferromagnetic MEMS (e.g., MEMS magnetometers, reconfigurable frequency-selective surfaces, and implantable magnetic microactuators for biomedical applications), nanomagnetomechanical systems, chemical sensors, micromachined sensors for plasma research, wireless sensor networks, microprobes for Parkinson's disease research, implantable and flexible 3-D microelectrode arrays, dynamic modeling of 3-D microelectrodes, microelectrode arrays for high-density neural stimulation and retinal prostheses, simulating the synthetic vision provided by retinal prostheses, micromachined systems to manipulate individual cells and to perform high-impedance patch-clamp recording, microactuators to solve the hydrocephalus shunt-clogging problem, wireless neural transceivers, brain-computer interfaces, and developing neural-control strategies and systems for functional deficits (e.g., spinal cord injury, ocular motility, and deep-brain stimulation). Lastly, he is dedicated to developing and improving graduate-level training programs in the multidisciplinary engineering fields of MEMS, nanotechnology, and neuroengineering. 